\title{
Cryogenic Transmission Electron Microscopy (Cryo-TEM) Reveals Morphological Changes of Liposomal Doxorubicin during In Vitro Release
}

Yong $\mathrm{Wu}^{1}$, Peter Petrochenko ${ }^{1,2}$, Francis C. Szoka ${ }^{3}$, Soumyarwit Manna ${ }^{1,2}$, Bonhye Koo ${ }^{1,2}$, Nan Zheng ${ }^{2}$, Wenlei Jiang ${ }^{2}$ and Jiwen Zheng ${ }^{1}$

1. Division of Biology, Chemistry and Materials Science, Office of Science and Engineering

Laboratories, Center for Devices and Radiological Health, Food and Drug Administration, Silver Spring, MD, United States

2. Office of Research Standards, Office of Generic Drugs, Center for Drug Evaluation and Research, Food and Drug Administration, Silver Spring, MD, United States

3. School of Pharmacy, University of California, San Francisco, CA

According to the draft guidance on doxorubicin hydrochloride liposome injections, US Food and Drug Administration (FDA) recommends for the in vitro liposome characterization of a test product and the reference listed drug (RLD) to be replicated with at least three lots. It is recommended to determine liposome morphology and number of lamellae since the degree of lamellarity affects drug loading, drug retention, as well as drug release rate from the liposomes. Furthermore, the physical state of the lipid bilayer and encapsulated doxorubicin should be investigated under a range of physiological conditions during in vitro drug leakage testing. However, currently the morphological changes of liposomal doxorubicin during in vitro drug release tests have not been thoroughly investigated.

In this study, we conducted a single-vial in vitro release test of doxorubicin liposome injections and evaluated their morphological changes during drug release. Two different in vitro release conditions, such as physical (10 degree rotation without $\mathrm{N}$-methylmorpholine) and chemical stress (210 orbital shaker with N-methylmorpholine), were investigated; morphology of liposomal doxorubicin was subsequently compared before and after drug release. Through rapid freezing, cryogenic transmission electron microscopy (cryo-TEM) was used to effectively preserve the native structure of liposomes in thin vitrified ice and provide a true snapshot of liposome morphology in nanometer resolution. The characteristic coffee-bean shape lipid vesicles were observed in the control liposomal doxorubicin sample, with precipitated doxorubicin drug encapsulated within the liposome (Fig. 1A). Under physical stress, larger unilamellar vesicles were identified which were possibly reconstructed from the lipids after release of doxorubicin from the liposomes (Fig. 1B). The remaining doxorubicin liposomes retained their integrity and their morphology appeared similar to the control. Under chemical stress, the coffeebean shaped liposomes gradually swelled and appeared spherical (Fig. 1C). In some liposomes, doxorubicin drug precipitates were not observed, indicating that the drug had been released as a result of chemical stress.

The results show cryo-TEM is a valuable tool for evaluating the critical morphological attributes of liposomes and monitoring morphological changes of doxorubicin liposome will help understand the release mechanism of doxorubicin under different conditions. 


\section{References:}

[1] Y Wu et al, International Journal of Pharmaceutics 505 (2016), p. 167.

[2] Y Wu et al, Microscopy and Microanalysis 20 (2014), p. 1348.

[3] Y Wu et al, Microscopy and Microanalysis 20 (2014), p. 1228.
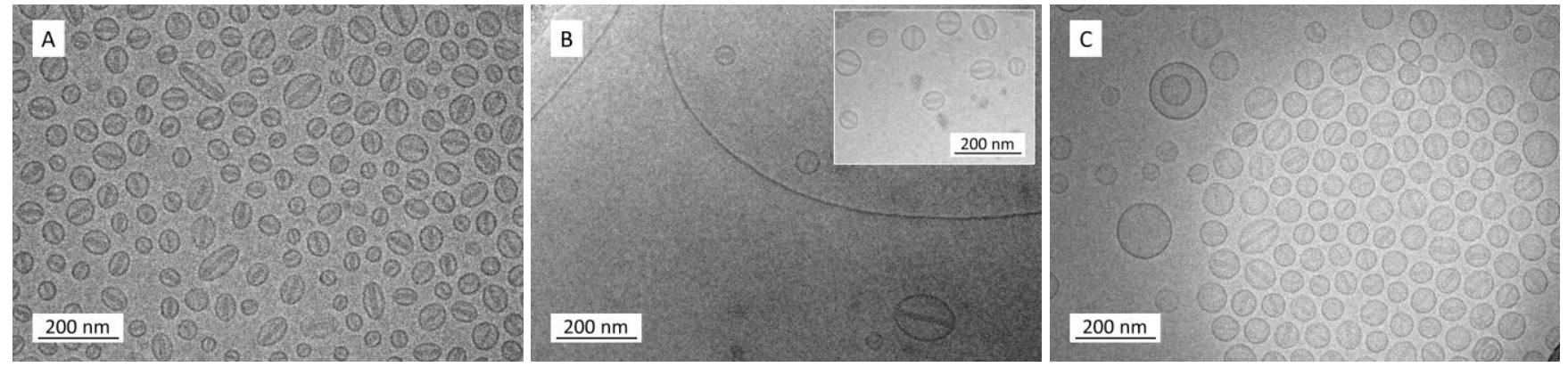

Figure 1. Representative cryo-TEM micrographs of A) control doxorubicin liposome, B) doxorubicin liposome under physical stress-induced release and C) doxorubicin liposome under chemical stressinduced release. 